\title{
A theoretical study of different carbon coatings effect on the depolarization effect and electrochemical performance of $\mathrm{LiFePO}_{4}$ cathode
}

\author{
Tao Liu ${ }^{\mathrm{a}, \mathrm{b}}$, Fengting $\mathrm{Cao}^{\mathrm{a}}$, Lingxiao Ren ${ }^{\mathrm{c}}$, Xichao $\mathrm{Li}^{\mathrm{a}}$, Shimei Sun ${ }^{\mathrm{a}}$, Xiaolin Sun ${ }^{\mathrm{a}}$, Zhao Zang ${ }^{\mathrm{d}}$, \\ Quanhai $\mathrm{Niu}^{\mathrm{a}}$, Jianfei $\mathrm{Wu}^{\mathrm{a}}$,* \\ ${ }^{\text {a }}$ Qingdao Institute of Bioenergy and Bioprocess Technology, Chinese Academy of Sciences, Qingdao, 266101, PR China \\ ${ }^{\mathrm{b}}$ University of Chinese Academy of Sciences, Beijing, 100049, PR China \\ ${ }^{c}$ College of Chemical Engineering, China University of Petroleum, Qingdao, 266580, PR China \\ ${ }^{\mathrm{d}}$ College of Mechanical and Electrical Engineering, China University of Petroleum, Qingdao, 266580, PR China
}

\section{A R T I C L E I N F O}

\section{Keywords:}

Carbon coating

$\mathrm{LiFePO}_{4}$

Depolarization effect

Electrochemical performance

Lithium-ion battery

\begin{abstract}
A B S T R A C T
In this work, three categories of advanced carbon materials coated on current collector are investigated. The results indicate that carbon coating has a positive influence on the depolarization effect and electrochemical performance of $\mathrm{LiFePO}_{4}$ cathode, different carbon coatings have drastically different influences, high rates in particular. The depolarization effect and rate performance show the following order: Graphene nanosheets (GNs) > Carbon nanotubes (CNTs) > Activated carbons (ACs), and the differences of rate capacities among them become more and more obvious with increasing rates. Especially for $5 \mathrm{C}$, the discharge capacities values are $122 \mathrm{mAh} \mathrm{g}^{-1}, 114.8 \mathrm{mAh} \mathrm{g}^{-1}, 106.8 \mathrm{mAh} \mathrm{g}^{-1}$ and $49.2 \mathrm{mAh} \mathrm{g}^{-1}$ for LFP-GNs-Al, LFP-CNTs-Al, LFP-ACs-Al and LFP-Al, respectively. The enhancement is attributed to the carbon coating acting as a transport system of electron between cathode materials and current collector, resulting in reducing the contact impedance within the electrodes, thus providing a favorable balance between fast ion diffusion and increased electron transport.
\end{abstract}

\section{Introduction}

In the last few years, rechargeable lithium-ion batteries have occupied a quality position in the market of energy storage systems with increasing energy demand of automotive power and emerging environmental concerns [1]. It has been proved that cathode material often sets limitation on the performance of lithium-ion batteries, despite many lithium transition metal oxides and polyanionic compounds have been proved to be excellent cathode for lithium-ion batteries, such as $\quad \mathrm{LiNi}_{0.8} \mathrm{Co}_{0.15} \mathrm{Al}_{0.05} \mathrm{O}_{2}, \quad \mathrm{LiNi}_{0.5} \mathrm{Co}_{0.2} \mathrm{Mn}_{0.3} \mathrm{O}_{2}, \quad \mathrm{LiNi}_{0.6} \mathrm{Co}_{0.2} \mathrm{Mn}_{0.2} \mathrm{O}_{2}$, $\mathrm{LiFePO}_{4}$ and $\mathrm{LiMn}_{2} \mathrm{O}_{4}$ [2-5]. As one of the mainstream cathode materials in commercial application, the olivine-structured $\mathrm{LiFePO}_{4}$ (LFP) combines excellent cycle life, intrinsic thermal stability, environmental compatibility and low production cost, which have attracted a huge amount of interest in cathode material for lithium-ion batteries [6-9]. However, compared with conventional spinel-type $\mathrm{LiMn}_{2} \mathrm{O}_{4}$ and layered $\mathrm{LiNi}_{\mathrm{x}} \mathrm{Co}_{\mathrm{y}} \mathrm{Mn}_{1}-\mathrm{x}-\mathrm{y} \mathrm{O}_{2}$, one of the most obvious obstacles for the widespread adoption of $\mathrm{LiFePO}_{4}$ is poor rate performance (particularly $>3 \mathrm{C}$ ), which are related to the characteristics of low conductivity (approximately $10^{-9}$ to $10^{-10} \mathrm{~S} \mathrm{~cm}^{-1}$ ) and slow lithium ion diffusion (approximately $10^{-14}$ to $10^{-16} \mathrm{~cm}^{2} \mathrm{~s}^{-1}$ ) of LFP, thus hinder its wider scope of application in the field of fast charge and discharge $[10,11]$.

It is an enormous challenge to improve battery power and rate performance, numerous effective approaches have been employed to improve the capacity and rate performance of LFP, including nanosizing, cation doping, coating LFP particles with conductive material and the addition of conductive additive with outstanding conductivity $[12,13]$. Nannan Zhao and co-workers [14] have confirmed that reducing the average particle size of LFP can improve the rate performance significantly, which is due to the small particle size can shorten the diffusion distance of electron and $\mathrm{Li}^{+}$simultaneously, and thus resulting in a fast electron and $\mathrm{Li}^{+}$transfer in the inside of LFP particles. Ian D. Johnson and co-workers [15] synthesized Nb-doped LFP nanoparticles by using a pilot-scale continuous hydrothermal flow synthesis process, the electrochemical testing reveals that the rate performance of LFP greatly improves with $1.0 \mathrm{wt} \% \mathrm{Nb}$-doping content, which can be ascribed to distort the olivine lattice, markedly increased the electrical conductivity of the LFP and furthermore increased $\mathrm{Li}^{+}$transport by Nbdoping. Wei Wei and co-workers [16] investigated the effect of graphene wrapping on the rate performance of LFP, results indicated that a partial graphene wrapping on LFP could improve the rate performance,

\footnotetext{
* Corresponding author.

E-mail address: wujf@qibebt.ac.cn (J. Wu).
} 
due to graphene wrapping can not only provide effective conducting network but also leaves pathway for the $\mathrm{Li}^{+}$diffusion. Cheolwoong Lim and co-workers [17] investigated the effects of different packing densities on geometric and electrochemical characteristics of lithium cobalt oxide battery. The results proved that high packing density electrodes have narrower pore size distribution, but capacity and rate capability are improved greatly by the geometric and electrochemical analysis, which is mainly attributed to the improvement of electrical contact between conductive additive and cathode material. Despite the above methods have been proved to make great progress in the electrochemical performance, but the rate performance leaves much to be desired to meet the practical applications [18].

As we know, for a high rate performance LIB, not only fast $\mathrm{Li}^{+}$ transport channel but also effective electron conducting network is necessary to be effectively constructed in the LFP cathode. Therefore, there are varieties of carbon materials have been reported to improve electrochemical performance of LFP by reducing the polarization effect of cathode material, mostly about being conductive additive to construct an effective electronic conducting network, [1,19]. Taiqiang Chen [20] and co-workers used acetylene black (OD), carbon nanotubes (1D) and reduced graphene oxide (2D) as conductive additives to investigate different carbon materials influence the electrochemical performance of mesocarbon microbeads anodes for lithium ion batteries, indicating that diverse carbon materials have different impacts on the electrode performance, which are related to the electron conduction and morphology of conductive additive, and the "plane-to-point" mode of GO-based additives displayed more flexible conducting network than the "line-to-point" mode of carbon nanotubes and the "point-to-point" mode of acetylene [21]. However, efforts via carbon materials coated on current collector are paid less attention to improve the performance of LFP. There are few literatures so far focusing on the carbon materials coated on current collector, which is used to promote the depolarization effect of cathode materials. With best of our knowledge, there is no report concerning the influence of various advanced carbon material coated on current collector on the electrochemical performance of LFP. We for the first time, try to investigate the effects of different carbon coatings on the depolarization effect and electrochemical performance of LFP, We found that the polarization effect of LFP particles with advanced carbon material coating was sharply reduced, resulting in a much better rate performance than the case without coating, which is closely related to the electron transport efficiency of carbon materials. Graphene coating shows the greatest promotion for depolarization effect among individuals in the comparison group, resulting in the best performance in fast charging and discharging.

\section{Experimental}

\subsection{Experimental materials}

The carbon coating current collectors were fabricated as follows: Firstly, added some dried PVDF to NMP suspension in a beaker, the stirring was lasted for $5 \mathrm{~h}$ after this addition to dissolve the PVDF fully. Then carbon materials consisted of GNs, CNTs and ACs which all have been calcined at high temperature were added into NMP solution and vigorously stirred for $24 \mathrm{~h}$ to obtain the uniformly dispersed solution. After this, the above carbon material slurries were spread well-distributed on current collector with $5 \mu \mathrm{m}$ (The optimum thickness has been proved) by an automatic coating machine (Hefei Ke Jing Materials Technology Co., Ltd., China.), and then dried in an oven at $120^{\circ} \mathrm{C}$ for $6 \mathrm{~h}$. The above carbon materials coated on current collectors are labeled hereafter as Al-GNs, Al-CNTs and Al-ACs. A commercially available LFP active materials with an average particle size of 500-600 nm were purchased from Shenzhen Dynanonic Co., Ltd., graphene nanosheets (GNs are mixture of single-layer and multi-layer, the latter is dominant) and carbon nanotubes (CNTs) provided by Shenzhen SUSN Sinotech New Materials Co., Ltd. Active carbons (ACs), $\mathrm{N}$-methylpyrrolidone
(NMP), Super-P (SP) and polyvinylidene fluoride (PVDF) are purchased from Shenzhen Kejing Star Technological Co. LTD. The component of separator and electrolyte were microporous polyethylene and $\mathrm{LiPF}_{6}$ $(1.0 \mathrm{M}$ in a $1: 1 \mathrm{v} / \mathrm{v}$ dimethyl carbonate and ethylene carbonate mixture) respectively.

\subsection{Characterization}

The microstructure and morphology of cathode materials were measured on a Hitachi S-4800(Hitachi, Japan) with an acceleration voltage of $10 \mathrm{kV}$. Cyclic voltammetry (CV) with a scanning rate of $0.1 \mathrm{mV} \mathrm{s}^{-1}$ between 2.8 and $4.2 \mathrm{~V}$ and the electrochemical impedance spectra (EIS) with the range of $0.01 \mathrm{~Hz}$ to $100,000 \mathrm{~Hz}$ were measured on an a CHI750E B16283 electrochemical measurement system (China). All the above experimental conditions were carried out under ambient condition.

\subsection{Electrochemical characterization}

The electrochemical experiments were measured by using typical 2032-type coin cells which were assembled in an argon-filled glove box $\left(\mathrm{H}_{2} \mathrm{O} \leq 0.01 \mathrm{ppm}, \mathrm{O}_{2} \leq 0.01 \mathrm{ppm}\right)$, the lithium electrode was used as counter and reference electrodes in the half cell. $\mathrm{LiFePO}_{4}$ (LFP), polymers binder (PVDF) and Super P were mixed in NMP solvent with the weight ratio of 85:10:5. Then the electrode slurry was maintained under constant stirring at room temperature to obtain a good dispersion. Shown as Fig.1, the cathode slurry was then pasted onto a carbon coating current collector by an industrial coating machine (Hefei Ke Jing Materials Technology Co., Ltd., China.) and dried in a vacuum oven at $130{ }^{\circ} \mathrm{C}$ for $24 \mathrm{~h}$ (As Structure illustrations shown in Fig.1.). The charge and discharge capacities of cathode materials were recorded using a Land СT2001A charge-discharge instrument, China.

\section{Results and discussion}

The structural and surface morphology characterization of $\mathrm{LiFePO}_{4}$ cathode with different carbon coatings are provided in Fig. 2, the scanning electron microscope (SEM) images reveal that the carbon coating homogeneously distributes on the surface of current collector. Compared with the case of current collector with a smooth surface, the surface morphologies of samples with coating are porous and stretchy, which result in larger dendritic structure and higher surface area. Fig. $2 \mathrm{e}-\mathrm{h}$ present the SEM images of full cross section of samples, as shown in Fig. 2e, loose contact between the cathode material and current collector is clear and distinguishable, which could result in large polarization and thus reduced electronic transmission efficiency. However, a sandwich morphology is clearly identified from Fig. $2 \mathrm{f}-\mathrm{h}$, as schematically illustrated in Fig. 3. These carbon coatings are tight and elastic enough to attach $\mathrm{LiFePO}_{4}$ grains onto the surfaces of each film and prevent them from "loose contact" phenomenon [22]. Moreover, there was no obvious change in the morphology and structure of $\mathrm{LiFePO}_{4}$ cathode with carbon coatings after 100 cycles at $1.0 \mathrm{C}$ rate (as showed in Fig. 2i-1). These are beneficial to strengthen the cohesiveness between the $\mathrm{LiFePO}_{4}$ grain and the current collector, as well as collect the micro-current of active substance during battery operation.

The electrochemical measurements and polarizability are carried out to evaluate the enhancement of electrochemical performance of $\mathrm{LiFePO}_{4}$ cathode with different carbon coatings. Herein, typical chargedischarge curves of LFP-Al, LFP-ACs-Al, LFP-CNTs-Al and LFP-GNs-Al at the $0.1 \mathrm{C}$ rate are shown in Fig.4a-d. The specific capacities were calculated based on including the mass of carbon in LFP/C [23] (the content of carbon coating is approximately $1-2 \%$ ). All the charge/discharge curves have a steady discharge plateau, LFP-Al, LFP-ACs-Al, LFP-CNTs-Al and LFP-GNs-Al delivered a specific capacity of $152.8 \mathrm{mAh} \mathrm{g}^{-1}, 154.9 \mathrm{mAh} \mathrm{g}^{-1}, 155.5 \mathrm{mAh} \mathrm{g}^{-1}$ and $155.9 \mathrm{mAh} \mathrm{g}^{-1}$ at the $0.1 \mathrm{C}$ rate with a voltage plateau near $3.4 \mathrm{~V}$, respectively. The 


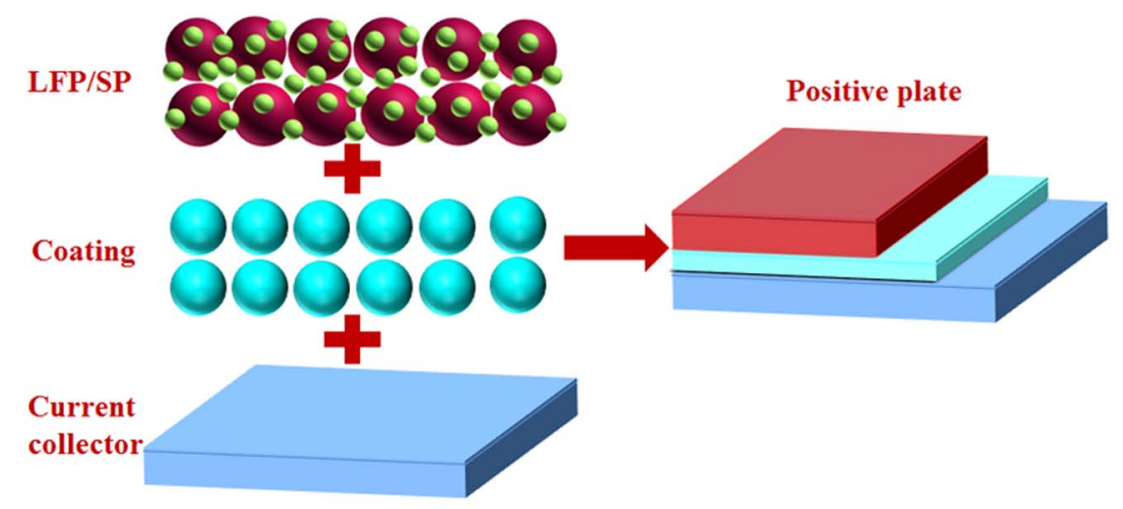

Fig. 1. Structure illustrations of $\mathrm{LiFePO}_{4}$ cathode with different carbon coatings.

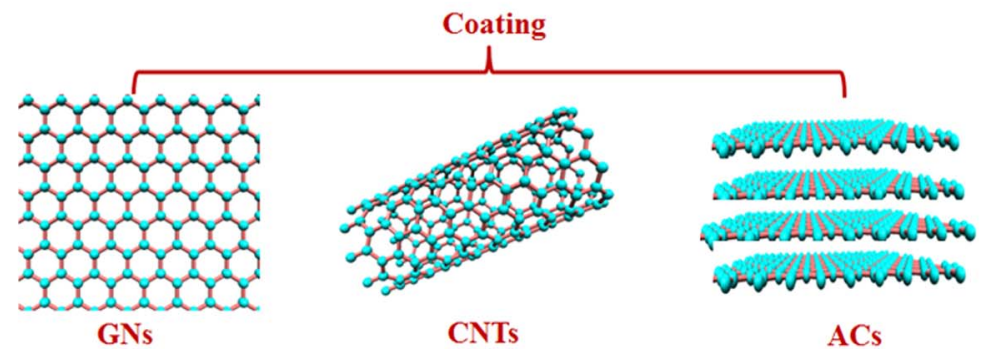

values of samples with carbon coatings are all close to the theoretical level and slightly larger than that of LFP-Al case. This is due to the fact that the current is too low to fully demonstrate polarization at low rate. However, at high rates (as shown in Fig.4e-h), for which the problem of polarization becomes prominent in the rate performance. It can be seen that the specific capacities of LFP-ACs-Al (128.2 $\left.\mathrm{mAh} \mathrm{g}^{-1}\right)$, LFP-CNTs$\mathrm{Al}\left(133.5 \mathrm{mAh} \mathrm{g}^{-1}\right)$ and LFP-GNs-Al (137.2 $\left.\mathrm{mAh} \mathrm{g}^{-1}\right)$ are significantly higher than that of LFP-Al $\left(98.6 \mathrm{mAh}^{-1}\right)$. Noticeable difference of the polarization between the charge and discharge plateau for different samples at both rates could be clearly observed. The polarization value of LFP-Al, LFP-ACs-Al, LFP-CNTs-Al and LFP-GNs-Al are $117 \mathrm{mV}$, $90 \mathrm{mV}, 68 \mathrm{mV}$ and $52 \mathrm{mV}$ at the $0.1 \mathrm{C}$ rate, when the C-rate increases to $2 \mathrm{C}$, the values rise to $720 \mathrm{mV}, 395 \mathrm{mV}, 198 \mathrm{mV}, 156 \mathrm{mV}$, respectively. Suggesting the depolarization of the LFP are indeed improved significantly with carbon coating, and different carbon materials have drastically different influences, the difference in depolarization became distinctly obvious with the increase of rates. Graphene exert the greatest promotion for depolarization, carbon nanotubes take second place, activated carbon the last. From these results, we can deduce that the conductivity property of carbon material is strongly related to depolarization. This is due to carbon coating act as a transport system of electron can facilitate fast electron transfer between the cathode and current collector, resulting in weakening polarization which arises from slow electron transport on the two-phase contact surface. The lowest polarization of LFP-GNs-Al electrode demonstrates an enhanced electron transfer due to high electrical conductivity properties of graphene, as well as Carbon nanotubes.

It is worth mentioning that the rate performance is key point to spread the application of LFP cathode material. The rate performance of all the samples from $0.1 \mathrm{C}$ to $5 \mathrm{C}$ is shown in Fig.5a. At the low current rates (0.1 C, $0.2 \mathrm{C}$ and $0.5 \mathrm{C}$ ), LFP-ACs-Al, LFP-CNTs-Al, LFP-GNs-Al and LFP-Al exhibit the similar high specific capacity, obvious differences among each other are not able to be observed in these low rates. However, different phenomenon occur in the high current rates $(1 \mathrm{C}$, $2 \mathrm{C}$ and $5 \mathrm{C}$ ), especially in $5 \mathrm{C}$, the capacities of LFP-ACs-Al, LFP-CNTs$\mathrm{Al}$ and LFP-GNs-Al are $106.8 \mathrm{mAh} \mathrm{g}^{-1}, \quad 114.8 \mathrm{mAh} \mathrm{g}^{-1}$ and $122 \mathrm{mAh}^{-1}$, respectively, much higher than that for LFP-Al (49.2 $\mathrm{mAh} \mathrm{g}^{-1}$ ), indicating that the rate performance show the following order: Graphene nanosheets $(\mathrm{GNs})>$ Carbon nanotubes (CNTs) > Activated carbons (ACs) > Current collector (AF), and the difference of capacity among them becomes more and more obvious with increasing rates, which is well consistent with the above results of polarization. The high rate capability of LFP cathode using carbon coated current collector is attributed to depolarization which could reduce localized collection of electron and improve electron transfer efficiency, particularly for high rate performance. Furthermore, the good cycle performance was also observed at the $1 \mathrm{C}$ rate as shown in Fig.5b. The LFP-ACs-Al, LFP-CNTs-Al and LFP-GNs-Al electrodes are extremely stable with no observable capacity loss even over 100 cycles, but instability and significant capacity fade occur to LFP-Al electrodes, we believe that carbon coating are crucially responsible for improving the cycle performance, this is mainly up to contribution of depolarization by carbon coating during battery operation.

Owing to the reversibility and the thermodynamics of the electrochemical reaction could be identified by the cyclic voltammetric technique [24]. The electrochemical behavior of LFP cathode with different carbon coating was evaluated by $\mathrm{CV}$ analysis at a scanning rate of $0.1 \mathrm{mV} \mathrm{s}^{-1}$. For a better comparison, the CV profiles of LFP-Al, LFPACs-Al, LFP-CNTs-Al and LFP-GNs-Al in the voltage range of 2.7-4.2 V at the 3rd scanning cycle were overlain as shown in Fig.6. All profiles exhibit one pair of symmetric cathodic (discharge) and the anodic (charge) peaks, which correspond to the re-intercalation and de-intercalation process in the electrodes. The potential interval between the reduction and oxidation peaks of LFP cathode with different carbon coatings show the following order: LFP-Al (0.443 V) > LFP-ACs-Al $(0.402 \mathrm{~V})>$ LFP-CNTs-Al $(0.269 \mathrm{~V})>$ LFP-GNs-Al $(0.227 \mathrm{~V})$, the polarization value of LFP-GNs-Al is obviously smaller than that of LFPCNTs-Al and much smaller than that of LFP-ACs-Al and LFP-Al, the results are well in accord with the electrochemical tests, suggesting that the depolarization are facilitated significantly by advanced carbon materials, and graphene have a great advantage over traditional carbon materials (ACs) in depolarization, as well as carbon nanotubes. These results indicate that carbon coated on current collector can markedly reduce the contact resistance between the LFP cathode and the current collector and decrease the polarization resistance of the cathode, which is due to significant improvement of the electronic conductivity between the cathode materials and the current collector by the excellent electrical conductivity of advanced carbon materials.

Electrochemical impedance spectroscopy (EIS) was carried out to further understand the electrodes reaction kinetics and $\mathrm{Li}^{+}$diffusion in 

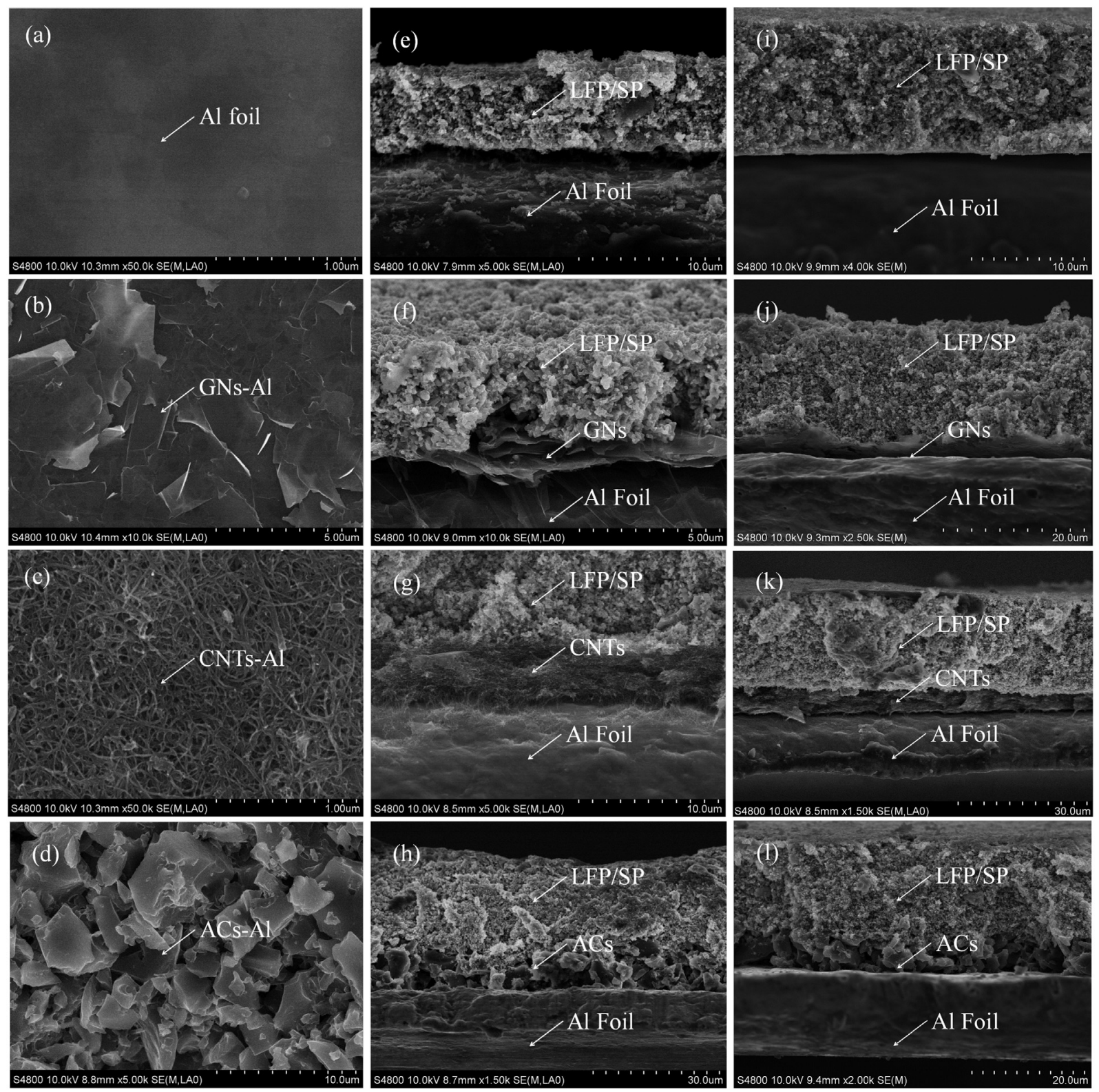

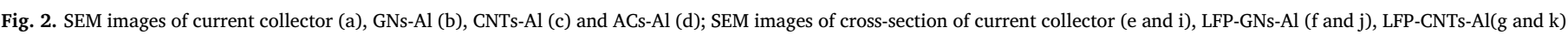
and LFP-ACs-Al(h and l), before and after 100 cycles respectively.

different systems. As the measured Nyquist plots and corresponding equivalent circuit model displayed in Fig.7. All the curves consist of three well-defined parts, an intercept at the real axis in the ultra-high frequency region is attributed to the ohmic resistance of the electrode system $\left(R_{\mathrm{o}}\right)$, which is corresponding to the sum of contact resistance between cathode film and current collector and the resistance of current collector, electrodes, electrolyte and separator [24], a partially overlapped semicircle in high and middle frequency range, related to the charge transfer resistance $\left(R_{\mathrm{ct}}\right)$ and an slope line under the low frequency region represents the Warburg impedance $\left(Z_{\mathrm{w}}\right)$, corresponding to the lithium ion diffusion in the active materials $[25,26]$. The simulation results of $R_{\mathrm{o}}$ and $R_{\mathrm{ct}}$ for the electrodes are listed in Table 1 . Owing to electrodes, electrolyte and separator also have a great influence on the ohmic resistance $\left(R_{\mathrm{o}}\right)$, which results in no obvious reduction in ohmic resistance $\left(R_{\mathrm{o}}\right)$ could be observed in this experiment. As far as $R_{\mathrm{ct}}$ is concerned, the $R_{\mathrm{ct}}$ of LFP-GNs-Al electrode $(74.7 \Omega)$ is the
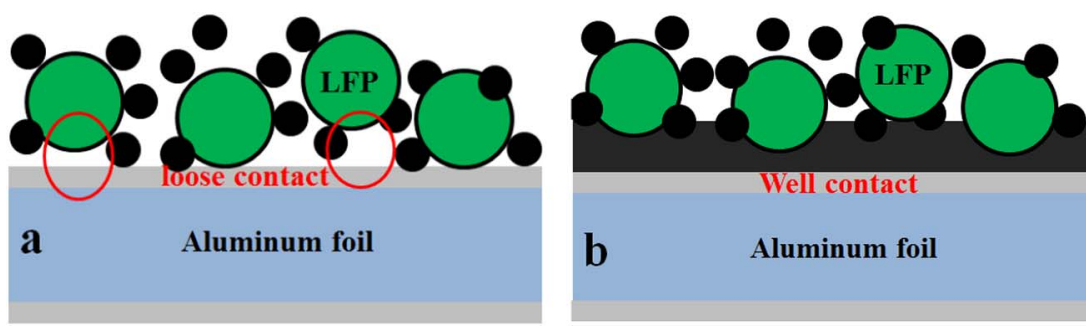

Fig. 3. Schematic of current collector without carbon coating(a) and with carbon coating(b) 

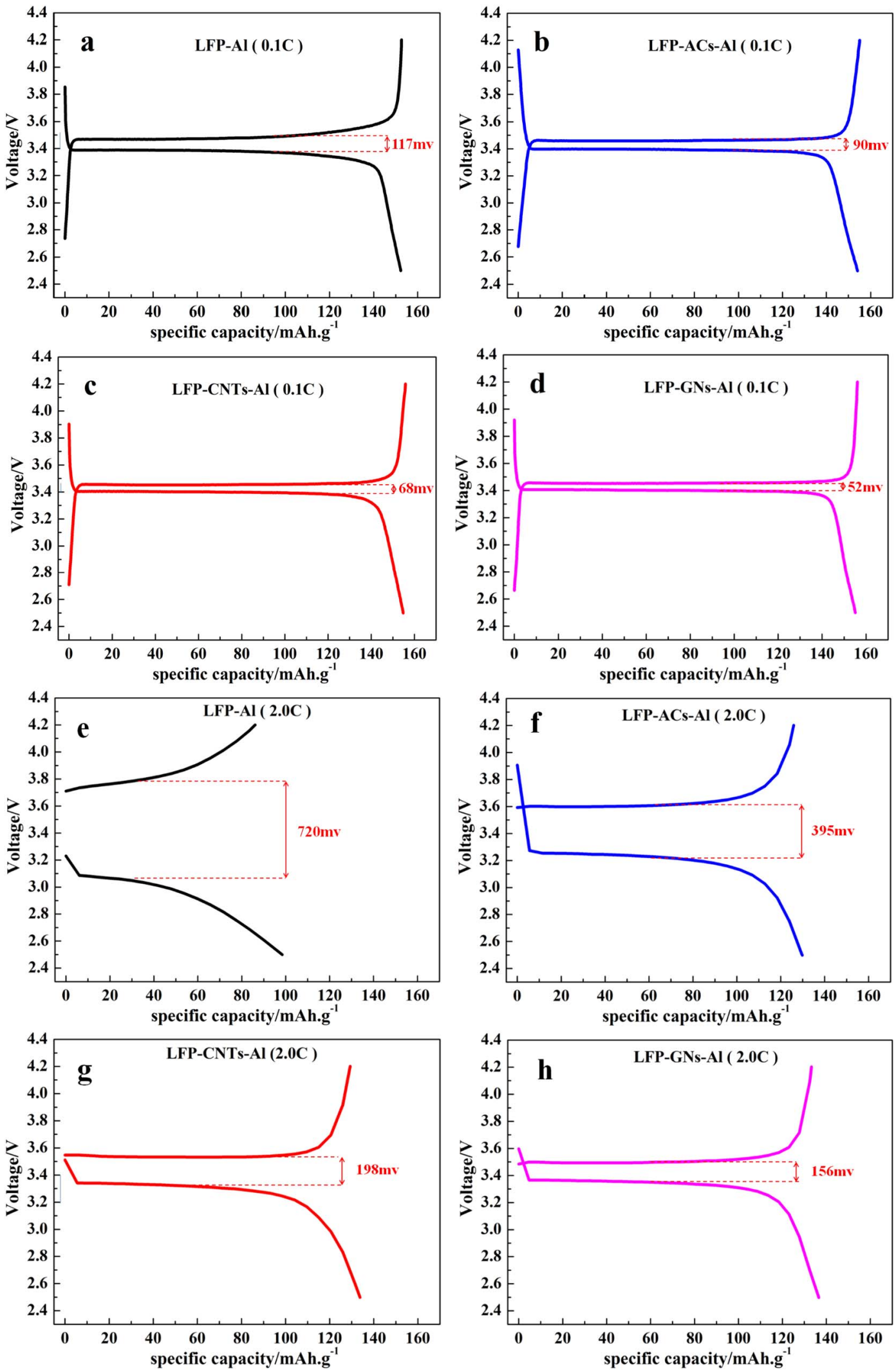

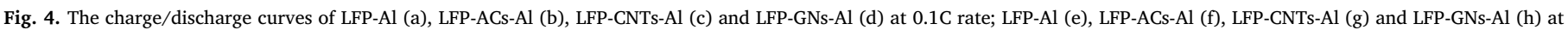
$2.0 \mathrm{C}$ rate.

lowest value and much smaller than that of other systems, and LFPCNTs-Al electrode $(118.3 \Omega)$ is the second-lowest value, and that of LFP-ACs-Al and LFP-Al are $231.4 \Omega$ and $482.8 \Omega$, indicating that the LFP cathodes using carbon coating promote charge transfer greatly, which enhances the electrochemical reaction in the cathodes, the results are also in good accordance with the electrochemical tests.

To deeply understand the significantly enhancement of rate performance of LFP with different carbon coatings, we calculated lithium 

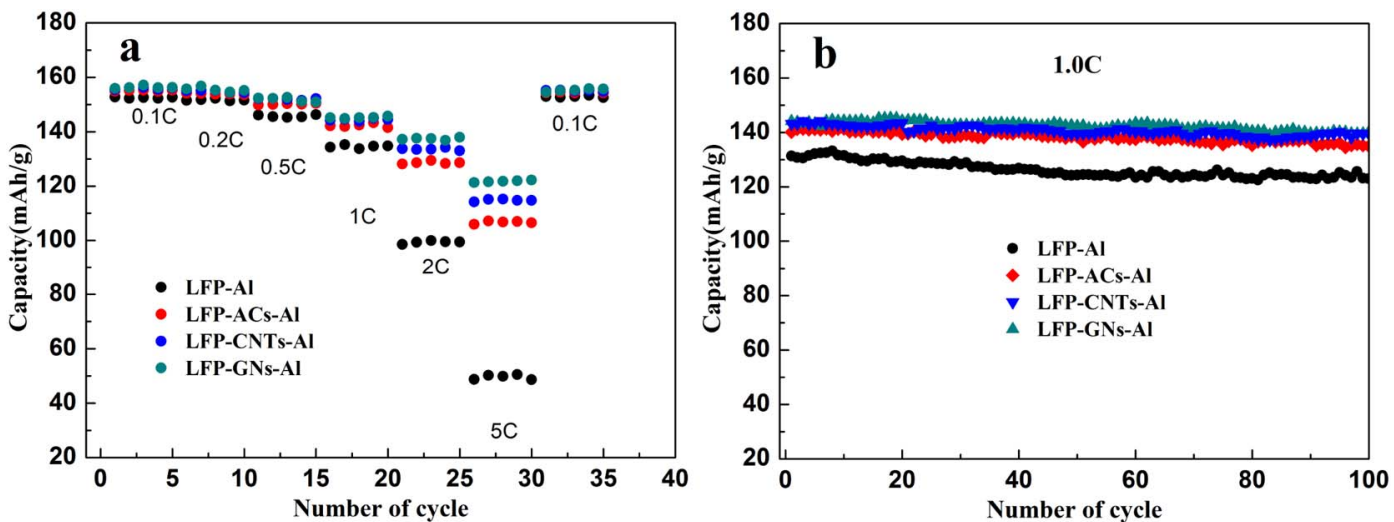

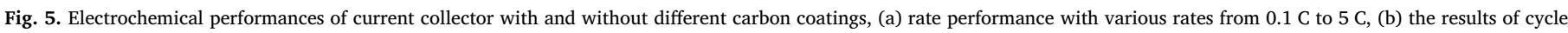
performances at the $1 \mathrm{C}$ rate.

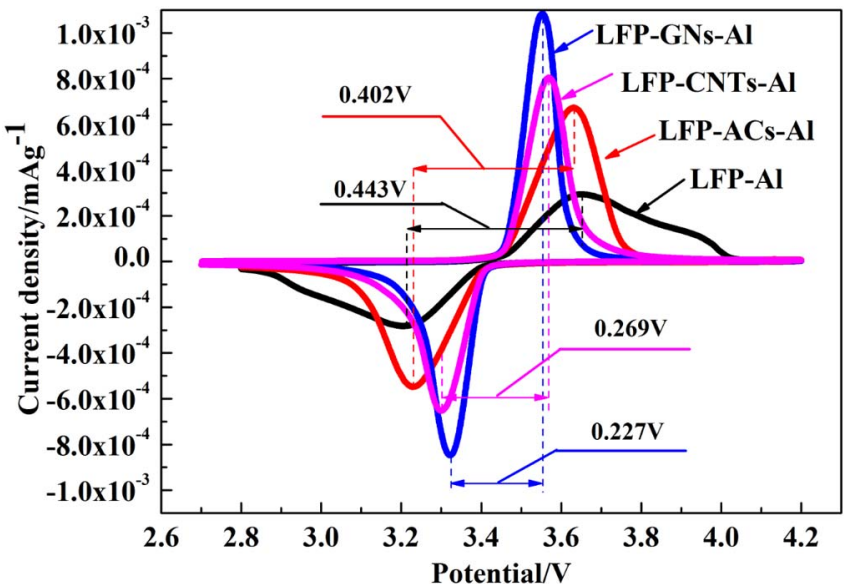

Fig. 6. Cyclic voltammograms for LFP cathode with different carbon coatings at a scanning rate of $0.1 \mathrm{mV} \mathrm{s}^{-1}$ under ambient temperature.

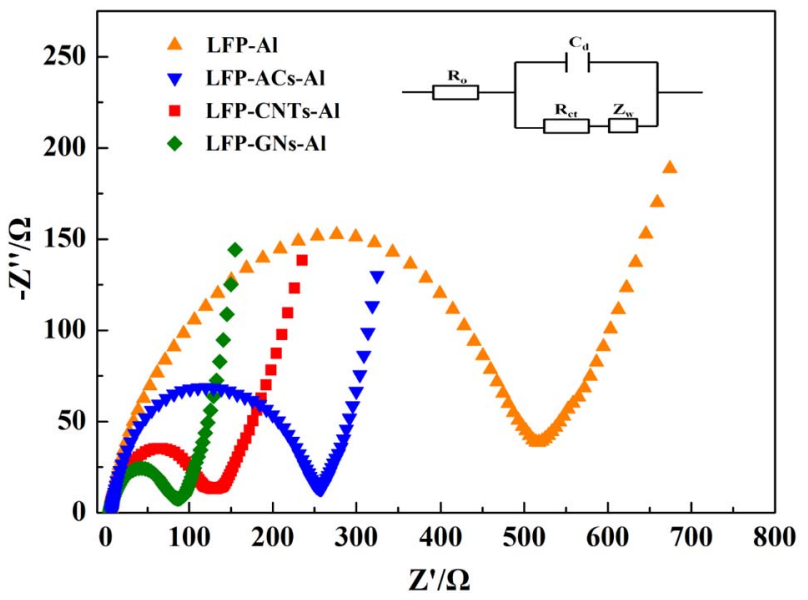

Fig. 7. Electrochemical impedance spectroscopy (EIS) of LFP cathode with different carbon coatings within the frequency range of $100 \mathrm{kHz}$ to $10 \mathrm{mHz}$.

Table 1

Impedance parameters of the different samples.

\begin{tabular}{lllll}
\hline Sample & LFP-Al & LFP-ACs-Al & LFP-CNTs-Al & LFP-GNs-Al \\
\hline$R_{\mathrm{o}}(\Omega)$ & 5.5 & 5.7 & 4.7 & 4.8 \\
$R_{\mathrm{ct}}(\Omega)$ & 482.8 & 231.4 & 118.3 & 74.6 \\
$D\left(\mathrm{~cm}^{2} \mathrm{~s}^{-1}\right)$ & $9.13 \times 10^{-13}$ & $2.48 \times 10^{-12}$ & $2.57 \times 10^{-12}$ & $6.0 \times 10^{-12}$ \\
$i^{0}\left(\mathrm{~mA} \mathrm{~cm}^{-2}\right)$ & $5.33 \times 10^{-5}$ & $1.11 \times 10^{-4}$ & $2.18 \times 10^{-4}$ & $3.46 \times 10^{-4}$ \\
\hline
\end{tabular}

ion diffusion coefficient $(D)$ by Eq. (1) $[23,27]$, and the exchange current density can be obtained by Eq.(2) [28].

$D=\frac{R^{2} T^{2}}{2 A^{2} n^{4} F^{4} C^{2} \sigma^{2}}$

$i^{0}=\frac{R T}{n F R_{c t}}$

Where $R$ is gas constant, $T$ is the temperature under operating conditions, $A$ stands for the surface area of the cathode, $n$ is related to the number of electrons in the process of oxidation-reduction reaction, $F$ is Faraday's constant, $C$ is molar concentration of lithium ion, the Warburg factor $(\sigma)$ can be obtained by Eq. (3), and the relationship between the $Z_{\mathrm{re}}$ and $\sigma$ at low frequencies is shown in Fig.8. All the data obtained from EIS are recorded in Table 1.

$Z_{r e}=R_{o}+R_{c t}+\sigma \omega^{-0.5}$

It is easily found that the exchange current density and lithium ion diffusion coefficient are greatly ameliorated by using carbon coating, indicating that depolarization which arise from carbon coating not only promote electron transfer efficiency, but also heighten lithium ion diffusion coefficient, thus providing a favorable balance between fast ion diffusion and increased electron transport, which results in showing the better rate performance, the higher speed electron transmission of carbon materials the greater promotion for the exchange current density and lithium ion diffusion coefficient. This result is also in agreement with the above electrochemical test.

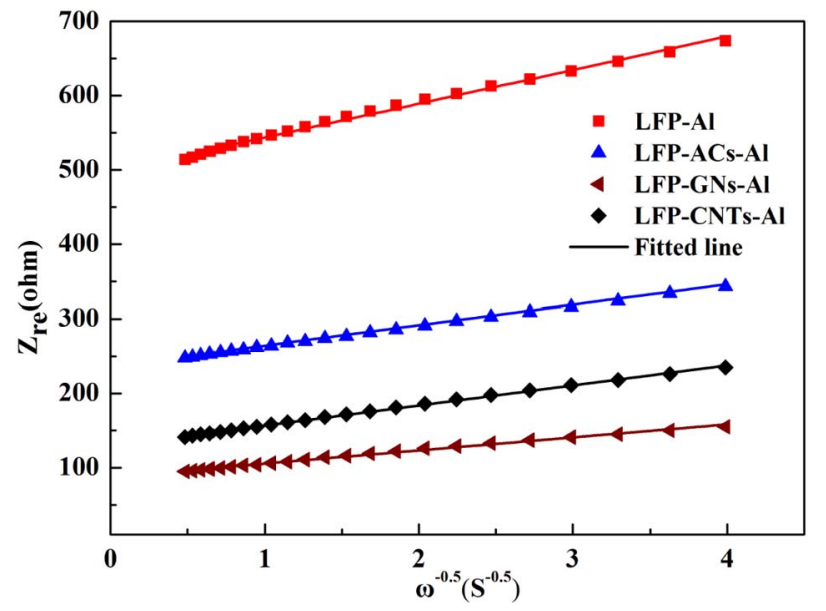

Fig. 8. The relationship between $\omega^{-0.5}$ and $Z_{\text {re }}$ at the low frequency for LFP-Al, LFP-ACs$\mathrm{Al}$, LFP-CNTs-Al, LFP-GNs-Al electrodes. 
This is a layer of passive film which consists of $\mathrm{Al}_{2} \mathrm{O}_{3}$ and $\mathrm{AlF}_{3}$ on the surface of current collector, resulting in holding back electron transport gravely which lead to large polarization between the cathode and current collector. Carbon materials coated on current collector play a crucial role in depolarization effect. Firstly, carbon coating can strengthen the cohesiveness between the $\mathrm{LiFePO}_{4}$ grain and the current collector, which is beneficial to collect the micro-current of active substance during battery operation. Secondly, carbon coating can reduce localized collection of electron which is the main reason for polarization, and thus improve electron transfer efficiency. The higher electron transport efficiency of carbon materials the more reduction for polarization between the cathode and current collector.

It is generally accepted that electrochemical performance bears great relation to electron conduction and lithium ion transportation, in a complete electrochemical processes, electrons and $\mathrm{Li}^{+}$should reach the same electrochemically active area simultaneously [25], owing to that the depolarization could provide a favorable balance between fast ion diffusion and increased electron transport, resulting in the better rate performance for LFP cathodes.

\section{Conclusions}

This work clearly demonstrates that the carbon materials coated on current collector play a crucial role in the depolarization effect and electrochemical performance of $\mathrm{LiFePO}_{4}$ cathode, and different carbon coatings have different effect on the high rate capability and cycle life of $\mathrm{LiFePO}_{4}$ cathodes, which is due to different enhancement of depolarization by different carbon materials. Graphene coating shows the greatest promotion for depolarization in the comparison group, resulting in outstanding performance in fast charging and discharging, as well as extremely long cycling life. The promotion of depolarization effect is closely related to the electron transport efficiency of carbon materials. This finding is of great importance for promoting the development of high-rate commercial lithium-ion battery.

\section{Acknowledgements}

This work was financially supported by National Natural Science
Foundation of China (No.21673267), Shandong Provincial Natural Science Foundation (No.ZR2015QZ01), Qingdao Postdoctoral Application Research Project, and Qingdao Industrial Energy Storage Technology Institute.

\section{References}

[1] C. Gong, Z. Xue, S. Wen, Y. Ye, X. Xie, J. Power Sources 318 (2016) 93-112.

[2] Y. Chen, Y. Zhang, B. Chen, Z. Wang, C. Lu, J. Power Sources 256 (2014) 20-27.

[3] W.M. Liu, Hu GR, Z.D. Peng, K. Du, Y.B. Cao, Q. Liu, Chin. Chem. Lett. 22 (2011) 1099-1102.

[4] X. Sun, X. Zhang, B. Huang, H. Zhang, D. Zhang, Y. Ma, J. Power Sources 243 (2013) 361-368

[5] J. Feng, Y. Wang, Appl. Surf. Sci. 390 (2016) 481-488.

[6] D.-T. Ngo, R. Scipioni, S.B. Simonsen, P.S. Jørgensen, S.H. Jensen, Int. J. Energy Res. 40 (2016) 2022-2032.

[7] C.-W. Ahn, J.-J. Choi, J. Ryu, B.-D. Hahn, J.-W. Kim, W.-H. Yoon, et al., Carbon 82 (2015) 135-142.

[8] J. Mun, H.-W. Ha, W. Choi, J. Power Sources 251 (2014) 386-392.

[9] X. Tian, Y. Zhou, X. Tu, Z. Zhang, G. Du, J. Power Sources 340 (2017) 40-50.

[10] A. Kuwahara, S. Suzuki, M. Miyayama, Ceram. Int. 34 (2008) 863-866.

[11] L. Ke, W. Lv, Su F-Y, Y.-B. He, C.-H. You, B. Li, et al., Carbon 92 (2015) 311-317.

[12] D. Wang, H. Li, S. Shi, X. Huang, L. Chen, Electrochim. Acta 50 (2005) 2955-2958.

[13] H.C. Shin, W.I. Cho, H. Jang, Electrochim. Acta 52 (2006) 1472-1476.

[14] N. Zhao, Y. Li, X. Zhao, X. Zhi, G. Liang, J. Alloys Compd. 683 (2016) 123-132.

[15] I.D. Johnson, E. Blagovidova, P.A. Dingwall, D.J.L. Brett, P.R. Shearing, J.A. Darr, J. Power Sources 326 (2016) 476-481.

[16] W. Wei, W. Lv, Wu M-B, Su F-Y, Y.-B. He, B. Li, et al., Carbon 57 (2013) 530-533.

[17] C. Lim, B. Yan, H. Kang, Z. Song, W.C. Lee, V. De Andrade, et al., J. Power Sources 328 (2016) 46-55.

[18] H. Kim, H. Kim, S.-W. Kim, K.-Y. Park, J. Kim, S. Jeon, et al., Carbon 50 (2012) 1966-1971.

[19] Y. Tang, F. Huang, H. Bi, Z. Liu, D. Wan, J. Power Sources 203 (2012) 130-134.

[20] T. Chen, L. Pan, X. Liu, Z. Sun, Mater. Chem. Phys. 142 (2013) 345-349.

[21] H. Bi, F. Huang, Y. Tang, Z. Liu, T. Lin, J. Chen, et al., Electrochim. Acta 88 (2013) 414-420.

[22] X. Hu, X. Sun, M. Yang, H. Ji, X. Li, S. Cai, et al., J. Mater. Sci. 52 (2016) 3597-3612.

[23] Y. Cui, X. Zhao, R. Guo, Electrochim. Acta 55 (2010) 922-926.

[24] P. Swain, M. Viji, P.S.V. Mocherla, C. Sudakar, J. Power Sources 293 (2015) 613-625.

[25] T. Liu, S. Sun, Z. Zang, X. Li, X. Sun, F. Cao, et al., RSC Adv. 7 (2017) 20882-20887.

[26] W.-M. Chen, L. Qie, L.-X. Yuan, S.-A. Xia, Hu X-L, W.-X. Zhang, et al., Electrochim. Acta 56 (2011) 2689-2695.

[27] X. Lei, H. Zhang, Y. Chen, W. Wang, Y. Ye, C. Zheng, et al., J. Alloys Compd. 626 (2015) 280-286.

[28] V.H. Nguyen, W.L. Wang, E.M. Jin, Gu H-B, J. Alloys Compd. 569 (2013) 29-34. 